\title{
Preparation and Characterization of a Novel Skin Substitute
}

\author{
Carlotta Castagnoli, Mara Fumagalli, Daniela Alotto, Irene Cambieri, Stefania Casarin, \\ Alessia Ostorero, Raffaella Casimiri, Patrizia Germano, Carla Pezzuto, and Maurizio Stella
}

Department of Plastic Surgery, Burn Unit and Skin Bank, Ospedale CTO, Turin, Via Zuretti 29, 10126 Torino, Italy

Correspondence should be addressed to Carlotta Castagnoli, ccastagnoli@hotmail.com

Received 20 May 2010; Revised 12 July 2010; Accepted 29 July 2010

Academic Editor: Anton M. Jetten

Copyright ( $) 2010$ Carlotta Castagnoli et al. This is an open access article distributed under the Creative Commons Attribution License, which permits unrestricted use, distribution, and reproduction in any medium, provided the original work is properly cited.

Autologous epidermal cell cultures (CEA) represent a possibility to treat extensive burn lesions, since they allow a significative surface expansion which cannot be achieved with other surgical techniques based on autologous grafting. Moreover currently available CEA preparations are difficult to handle and their take rate is unpredictable. This study aimed at producing and evaluating a new cutaneous biosubstitute made up of alloplastic acellular glycerolized dermis (AAGD) and CEA to overcome these difficulties. A procedure that maintained an intact basement membrane was developed, so as to promote adhesion and growth of CEA on AAGD. Keratinocytes were seeded onto AAGD and cultured up to 21 days. Viability tests and immunohistochemical analysis with specific markers were carried out at 7, 14, and 21 days, to evaluate keratinocyte adhesion, growth, and maturation. Our results support the hypothesis that this newly formed skin substitute could allow its permanent engraftment in clinical application.

\section{Introduction}

The burn patient is prone to a complex, articulated, and polymorphous clinical picture, based on a systemic inflammatory response, affecting multiorgan function, with a high mortality rate. As extensive burns generate large areas of necrotic skin, the therapeutic priority is the removal of the necrotic tissue, so as to limit the inflammatory response. Although autologous skin grafting is the gold standard for definitive wound coverage, it is difficult to find suitable donor areas in a patient with extensive burns $[1,2]$. Dermal skin substitutes may be used to handle the problem of donor site shortage when dealing with major skin loss. Indeed, recent developments in the multidisciplinary field of tissue engineering have yielded many novel tissue replacements and implementation strategies. Scientific advances in biomaterials, stem cell isolation, growth and differentiation factors, and biomimetic environments have created unique opportunities to produce tissues in the laboratory. However, although there have been enormous advances in the development of skin substitutes over the last 3 decades, there are still major obstacles to be overcome in the quest for an optimal skin substitute.
Successful treatment in burn care with dermal skin substitutes requires low antigenicity, the capacity for rapid vascularization and a stable dermal template. Even if, to date, the best cutaneous substitute remains alloplastic skin, it does not ensure a permanent reconstruction and tends towards rejection. For some decades this problem was solved by surgical techniques, based on a combination of tissue and/or cell auto/allografts [3-7]. Human alloplastic grafts were used to support cultivated autologous keratinocytes (CEA) in a clinical setting, offering a permanent wound coverage with the Cuono technique. However it has the drawback that the CEA take is not always reproducible, making it somewhat unpredictable [8-10].

The combination of technical difficulties including high costs, unpredictable graft take, and prolonged instability of the grafts has led researchers to carry out research in an effort to find more resilient substitutes. To this aim, donor skin allografts preserved in $85 \%$ glycerol are often used as a temporary coverage for large burn wounds, as glycerol does not affect the structural integrity of the skin [11]. Indeed, the glycerol-preserved skin allograft, although devitalised, does retain its morphological structure and, therefore, can be used as a temporary skin substitute, or 
grafted as a dermal template. Glycerol-preserved allografts, that have advantageous biomanual properties, are being investigated as scaffolds for tissue engineering applications. The aim of the research reported herein was an in vitro construction of a skin substitute made up of an alloplastic acellular glycerolized dermis (AAGD) scaffold directly seeded with low-density keratinocytes.

\section{Materials and Methods}

The study was carried out according to a protocol approved by the CTO/San Giovanni Battista Hospital Institutional Ethical Board (Turin, Italy prot. 0046054) and written informed consent was obtained from all patients included.

2.1. The Scaffold Preparation and De-Epithelialization. Donor skin grafts (about $20 \mathrm{~cm}^{2}$ ), preserved at $85 \%$ glycerol in the Turin Skin Bank and unfit for transplantation, were used as the skin substitute scaffold. The glycerol was removed from the grafts by sequential washing in sterile $0.9 \%$ saline solution at $+37^{\circ} \mathrm{C}$. The grafts were then incubated overnight at $+33^{\circ} \mathrm{C}$ under continuous shaking in RPMI 1640 (GibcoLife Technologies, Grand Island, NY, USA) and supplemented with $1 \%$ human albumin (Farma Biagini, Italy), Vancomycin $100 \mu \mathrm{g} / \mathrm{mL}$ (Vanco, Pulitzer Italiana srl; Roma, Italy), Imipenem $100 \mu \mathrm{g} / \mathrm{mL}$ (Tienam, Merck, Sharp and Dohme, Italy), sulfametoxazole-trimethoprim $50 \mu \mathrm{g} / \mathrm{mL}$ (Bactrim, Roche, Milan, Italy) and Fluconazole $50 \mu \mathrm{g} / \mathrm{mL}$ (Diflucan, Pfizer Italiana, Italy). The following day, the epidermal layer was removed by three different techniques: one using dispase II $\left(3 \mathrm{~h}\right.$ at $+37^{\circ} \mathrm{C}$ in $\left.5 \% \mathrm{CO}_{2}\right)$, another trypsin $\left(3 \mathrm{~h}\right.$ at $+37^{\circ} \mathrm{C}$ in $\left.5 \% \mathrm{CO}_{2}\right)$ and the third without the use of any enzyme, with only manual removal. The manual removal involved massaging the graft by hand, for an average of one minute for an allograft of about $20 \mathrm{~cm}^{2}$. This procedure was followed by manual stripping of the epidermal layer, which detached very easily. This detachment was facilitated by its overnight incubation and continuous shaking, as reported above. The whole procedure was simple and reproducible by any of the laboratory personnel (Figure 1).

2.2. Keratinocyte Cultures. Human split-thickness skin grafts $(0.4 \mathrm{~mm}$ thick) were obtained from healthy individuals. Serial enzymatic cell separation was used to obtain cell suspensions from these samples using $2 \mathrm{mg} / \mathrm{mL}$ dispase II (Roche Manheim, Germany) and trypsin 0.05\% (Invitrogen Life Technologies, USA). The cells, plated at $1.6 \times$ $10^{4} \mathrm{cells} / \mathrm{cm}^{2}$ density, were grown on a irradiated 3T3J2 layer, as described by Rheinwald and Green [12], in Dulbecco's Modified Eagle Medium (DMEM, Gibco, UK) and $10 \%$ fetal bovine serum (FBS, New Zealand origin) supplemented with $5 \mu \mathrm{g} / \mathrm{mL}$ insulin (Sigma-Aldrich, USA), $0.4 \mu \mathrm{g} / \mathrm{mL}$ hydrocortisone (Sigma-Aldrich, USA), $10^{-10} \mathrm{M}$ cholera toxin (Sigma-Aldrich, USA), $2 \times 10^{-10} \mathrm{M}$ triiodothyronin (Sigma-Aldrich, USA), $5 \mu \mathrm{g} / \mathrm{mL}$ apo-transferrin (Invitrogen Life Technologies, USA), $2 \mathrm{mM}$ glutamine (SigmaAldrich, USA), and $50 \mathrm{UI} / \mathrm{mL}$ penicillin-streptomycin antibiotic solution. The flasks were incubated at $+37^{\circ} \mathrm{C}$ in $5 \% \mathrm{CO}_{2}$.
At the first medium change (5 days after the initial plating), the medium was further supplemented with $10 \mathrm{ng} / \mathrm{mL}$ epidermal growth factor (Sigma-Aldrich, USA) and changed every 48-72 hours.

2.3. Preparation of Keratinocyte Suspension. Primary cultures of human keratinocytes were grown to approximately $80 \%$ confluence after about fourteen days. The cells were enzymatically separated by digestion with $0.05 \%$ trypsin (Invitrogen Life Technologies, USA). Residual 3T3-J2 were removed before harvesting by washing with $0.05 \%$ trypsin. The recovered keratinocytes were rewashed and resuspended in culture medium, at a density of $4 \times 10^{5}$ cells $/ \mathrm{mL}$. A syringe was fitted with a spray cap to nebulize $5 \mathrm{~mL}$ of these suspensions onto a de-epithelialized dermis $\left(20 \mathrm{~cm}^{2}\right)$, which were then incubated at $+37^{\circ} \mathrm{C}$ in $5 \% \mathrm{CO}_{2}$, for 21 days.

Serial enzymatic cell separation was used to obtain keratinocyte suspensions from the skin biopsy as reported above. They then were sprayed at $1.5 \times 10^{5} \mathrm{cells} / \mathrm{cm}^{2}$ onto the scaffold and the culture medium was changed every 2-3 days. The keratinocytes were nebulized onto the scaffold without a murine 3T3-J2 fibroblast feeder layer.

2.4. Proliferation Assay. Enzyme activity was assessed to define the skin substitute viability with an MTT test (Roche $\mathrm{GmbH}$, Mannheim, Germany) at 7, 14, and 21 days after spraying, as described by Alotto et al. [13]. Spectrophotometer readings were performed at $570 \mathrm{~nm}$, with an Eppendorf BioPhotometer.

2.5. Tissue Specimens and Staining Procedure. Two sixmillimetre punch biopsies were made at 7,14 , and 21 days after cell nebulization: one was snap frozen in isopentane and stored at $-70^{\circ} \mathrm{C}$, the other was included in paraffin for histological analysis. Frozen sections $(7 \mu \mathrm{m})$ were airdried, acetone fixed and incubated with primary mouse monoclonal antibodies (MoAb), followed by avidin-biotin, as previously described [14]. Staining was developed with 3-amino-9-ethyl carbazole as a chromogen and the sections were then counterstained with Mayer's hematoxylin solution and mounted with glycerol gelatine. The second MoAb was detected by the avidin-biotin-horseradish peroxidase system (DAKO, Milan, Italy). The mouse antihuman MoAb used in this paper included: CD3 (clone SK7; dilution 1:10; Becton Dickinson, USA), CD68 (clone EBM11; dilution 1:100; Dako, USA), CD83 (clone HB15a; dilution 1:20; Immunotech, France), cytokeratin (clone AE1/AE3; dilution 1:50; Dako, USA), cytokeratin 14 (clone LL002; dilution $1: 10$; Vector Laboratories, USA), cytokeratin 16 (clone LL025; dilution $1: 20$; Vector Laboratories, USA), E-cadherin (clone NCH-38; dilution 1:50 DakoCytomation, USA), laminin (clone 4C7; dilution 1:100; DakoCytomation, Denmark), laminin V (clone P3H9-2; dilution 1: 100; Chemicon International, USA), collagen IV (clone CIV 22; dilution 1:100; DakoCytomation, Denmark), collagen VII (dilution $1: 100$; Millipore, USA). Histological analysis was performed to confirm specimen adequacy. 


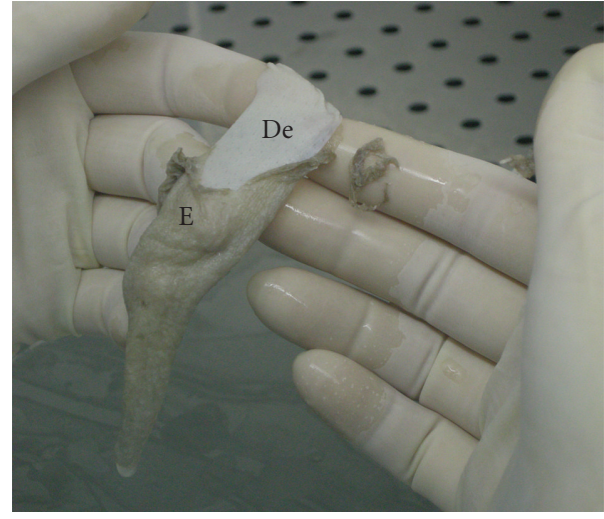

(a)

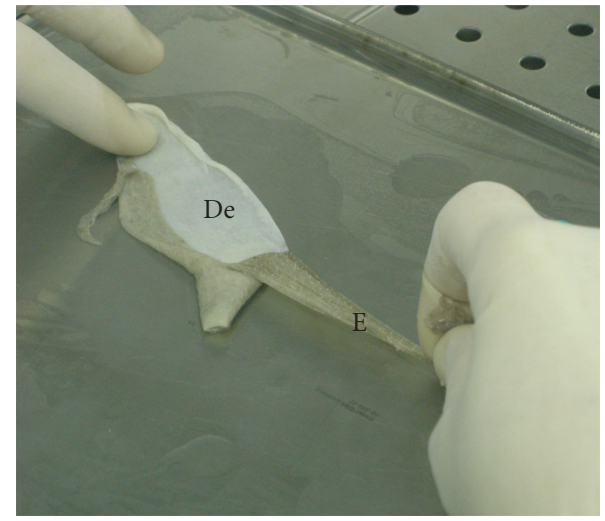

(b)

FIgURE 1: The manual removal involved massaging the graft by hand (a) followed by manual stripping of the epidermal layer, which detached very easily (b); E: epidermis, De: dermis.

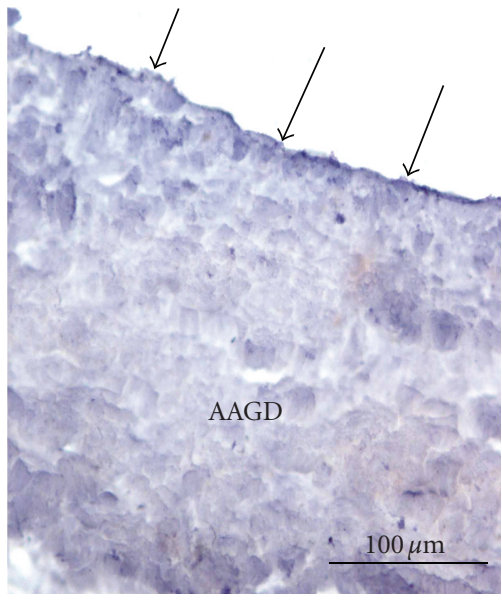

(a)

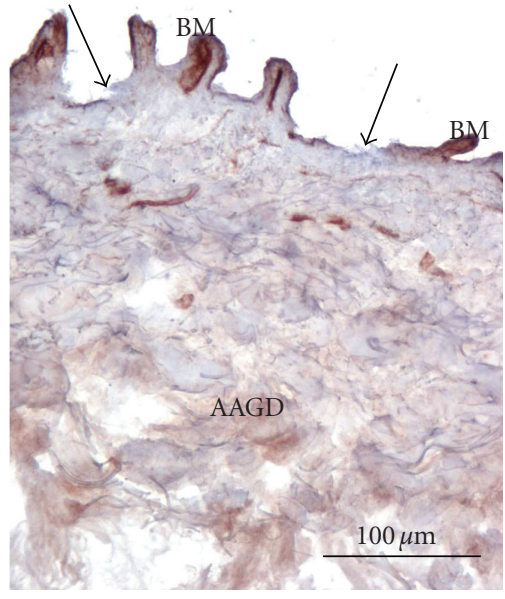

(b)

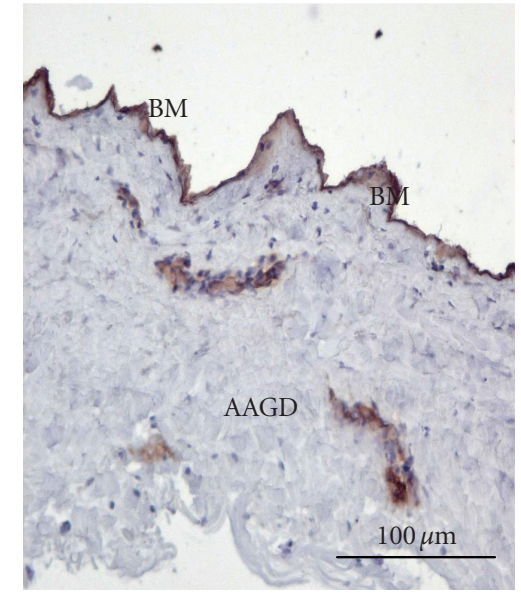

(c)

FIgURE 2: The dermis basement membrane obtained by the three different techniques stained with Collagen IV MoAb. (a) Dispase II, (b) Trypsin, (c) the manual method. Only the manual method left an integral structure. Arrows indicate the absence of basement membrane. Original magnification: 10x. AAGD: alloplastic acellular glycerolized dermis; BM: basement membrane.

2.6. Quantitative Analysis of Dermis Infiltrating Cells. Stained cells were counted in three sequential sections for each specimen, with separate consideration given to the subpapillary and reticular dermis. At least 6 series of three sequential sections were analysed for each biopsy and 8 high-power random fields analysed and counted for each section. The T cell subpopulation was evaluated by staining infiltrating cells with Moab antiCD3 molecule, whilst macrophages with antiCD68 MoAb and DDC population were detected with antiCD83 MoAb.

\section{Results}

3.1. The Scaffold Preparation. The glycerolized allografts were de-epithelialized with three different methods and used as a scaffold for keratinocyte growth and differentiation. The first method used dispase II, which cleaves the anchoring system between the dermis and epidermis. The second used trypsin, which breaks-down the single cells, producing a cellular suspension. The third was a manual method without the use of any enzyme. Specific markers and immunohistochemistry assessed the basement membrane of all the three types of dermis. The scaffold was then nebulized with keratinocytes and cell adhesion analysed by immunohistochemistry and histology.

3.1.1. The Basement Membrane. The presence of a basement membrane was assessed by immunohistochemical analysis on the three different preparations of AAGD scaffolds. Specific basement membrane glycoprotein monoclonal antibodies were directed against pan-laminin, laminin 5, collagen IV and VII were evaluated. Immunohistochemistry demonstrated that dispase II had completely removed the basement membrane in all sections analysed (Figure 2(a)). Trypsin had left only a few, incomplete basement membrane in limited areas of the dermis (Figure 2(b)). Conversely, the manual 


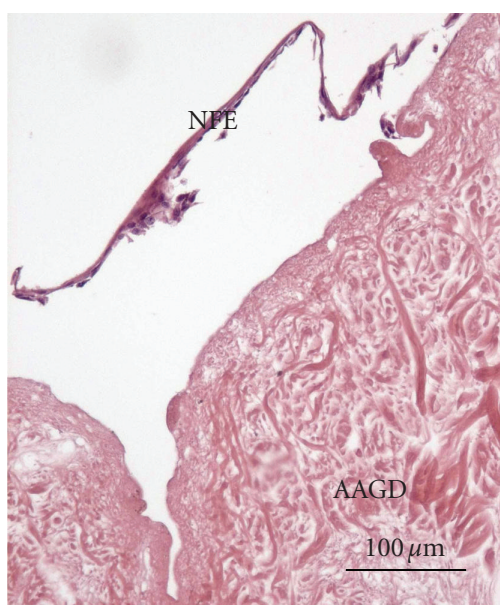

(a)

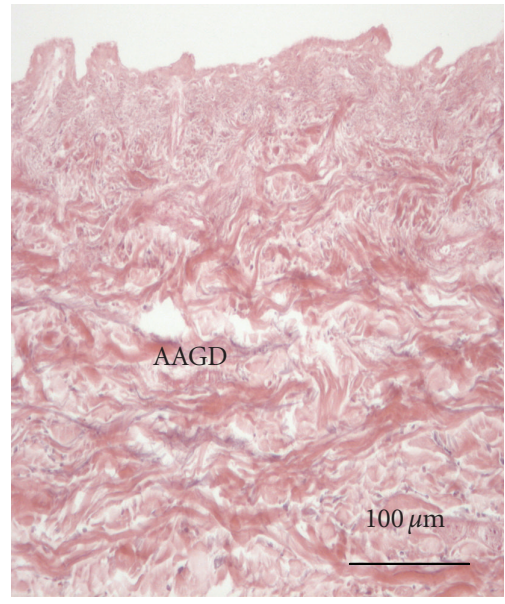

(d)

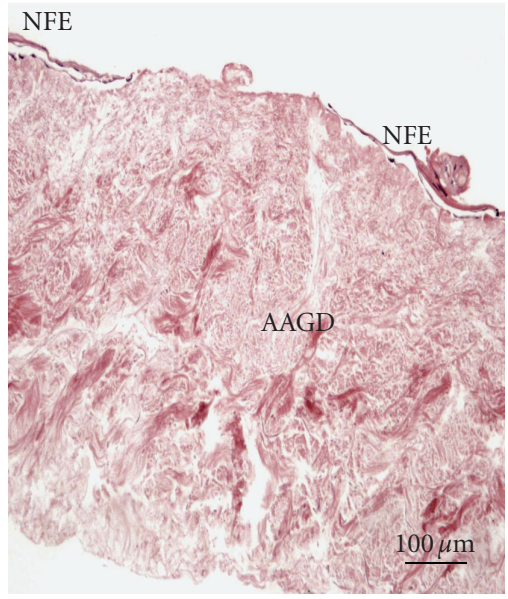

(b)

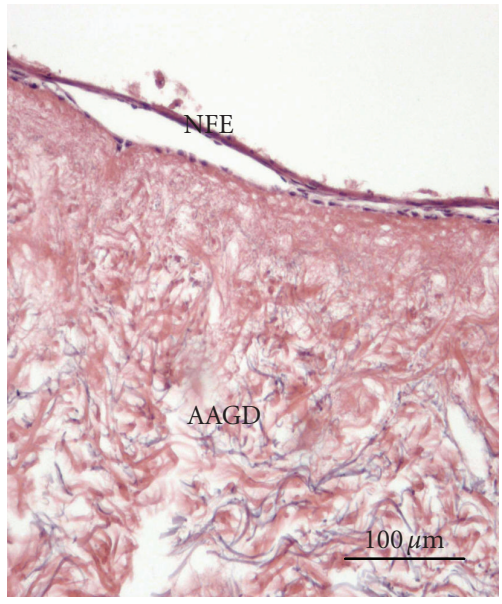

(e)

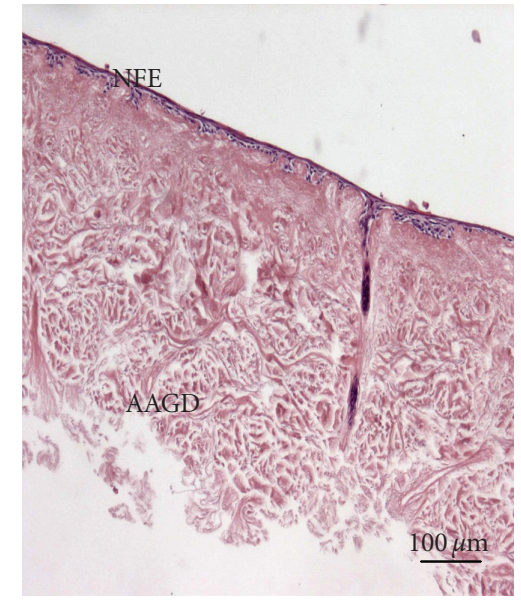

(c)

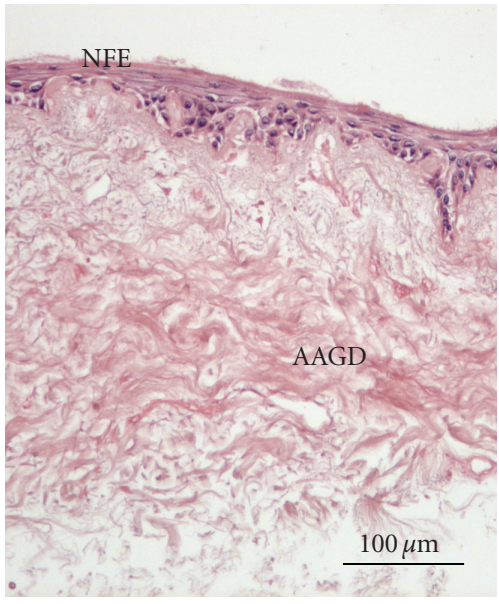

(f)

FIGURE 3: Histomorphological analysis of the dermis obtained by the three different techniques nebulized with keratinocytes. The sample refers to the 14th day ((a), (b), and (c)) and to the 21st day ((d), (e), and (f)), (a) and (d) Dispase II, (b) and (e) Trypsin, (c) and (f): the manual method. Nebulized cell adherence was observed only in the one prepared manually. Original magnification: 10x ((a), (d), (e) and (f) $5 \mathrm{x}$ ((b) and (c)). AAGD: alloplastic acellular glycerolized dermis; NFE: newly formed epithelium.

method had left the membrane intact throughout the dermis profile, as shown in Figure 2(c). Pan-laminin and laminin $5 \mathrm{MoAb}$ were positive in the whole basement membrane in all these specimens, with a strong collagen IV and VII positivity (data not shown).

3.1.2. Analysis of Keratinocyte Adhesion. The three types of dermis were nebulized with uncultured, or primary culture keratinocytes and analysed by histology at 7, 14, 21 days after cell seeding. After dispase II treatment, in the absence of basal membrane, the dermal sections had only a few cells attached to the alloplastic scaffold at 7 days (data not shown). Moreover, cell detachment was observed at two weeks, indicating poor cell adhesion to the scaffold (Figure 3(a)) indeed there were no cells at 21 days (Figure 3(d)). Those obtained with the trypsin method showed the presence of cellular foci on the dermis at 7 days, whilst there was a noncontinuous cellular monolayer at 14 (Figure 3(b)) and 21 days (Figure 3(e)).
The biosubstitute sections, obtained with the third manual method, showed one or two continuous cell layers at 7 days, at 14 there was a pluristratified epithelium (Figure 3(c)) and a differentiated epithelium with corneous layer at 21 days (Figure 3(f)). There was no detachment of keratinocytes from the basal membrane.

\subsection{The Scaffold Analysis}

3.2.1. AAGD Immunogenicity. The presence of immunocompetent cells in the AAGD tissue was determined by immunohistochemistry on the tissue sections. There was no staining with specific antibodies directed against $\mathrm{T}$ lymphocytes, macrophages, or dermal dendritic cells at 5 days of culture (data not shown). Histomorphological analysis showed the presence of nonviable infiltrating cells in the skin section at day 0 (Figure 4(a)), the cells decreased at 2 days (Figure 4(b)) and were negative as from 7 days, as demonstrated in Figures 4(c) and 4(d) 


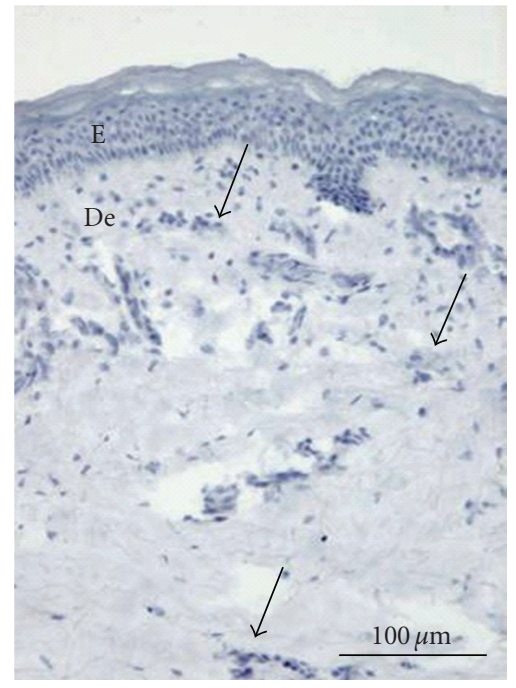

(a)

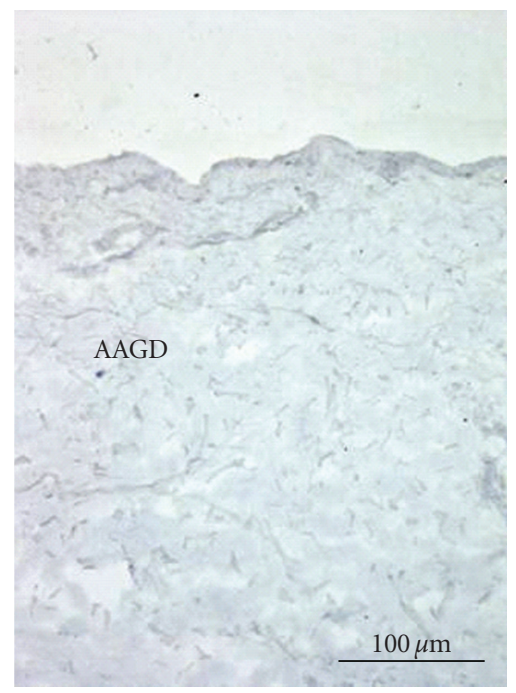

(c)

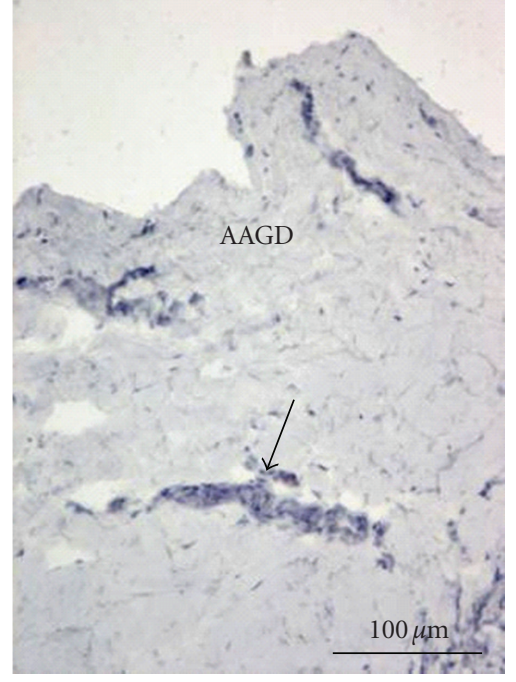

(b)

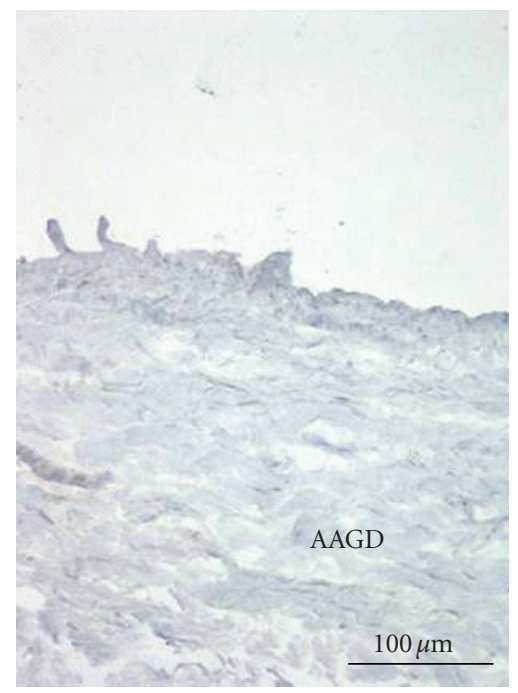

(d)

FIgURE 4: Histomorphological analysis performed on the de-epithelialized dermis to verify the integrity of the tissue architecture and determine the cell presence over time. Sections were stained with Mayer's hematoxylin solution. (a) at day 0, after the glycerol removal and before the de-epithelialization: arrows indicate the presence of infiltrating cells; (b) at 2 days, (c) at 7 days, (d) at 14 days. The number of ghost cells decrease constantly over time and are no longer present as from 7 days. Original magnification: 10x. E: epidermis, De: dermis, AAGD: alloplastic acellular glycerolized dermis.

were there was no staining of the nuclei at 7 or 14 days.

3.2.2. Assessment of the AAGD Viability. An MTT quantitative analysis was also carried out on the scaffold to determine the AAGD viability. The scaffold viability was compared to the fresh skin sample. The terms "fresh sample" indicate a specimen analysed immediately after harvesting and used as a positive control to describe the $100 \%$ viability activity in the MTT assay. The results confirm the immunohistochemistry data (Figure 5).
3.3. The Biosubstitute Analysis. As only the manual method left the basement membrane intact, it was selected and used for the preparation of the biosubstitute scaffold, that is, the object of this work (AAGD). Keratinocytes obtained, either directly from the biopsy (uncultured cells), or after in vitro primary culture, were seeded by means of nebulization onto the scaffold in the absence of a murine 3T3-J2 fibroblasts feeder layer. Quality control on the keratinocyte proliferation was carried out by the colony forming efficiency (CFE) technique, which was evaluated after 14 days of culturing with Petri dishes. All the nebulised samples showed at least 


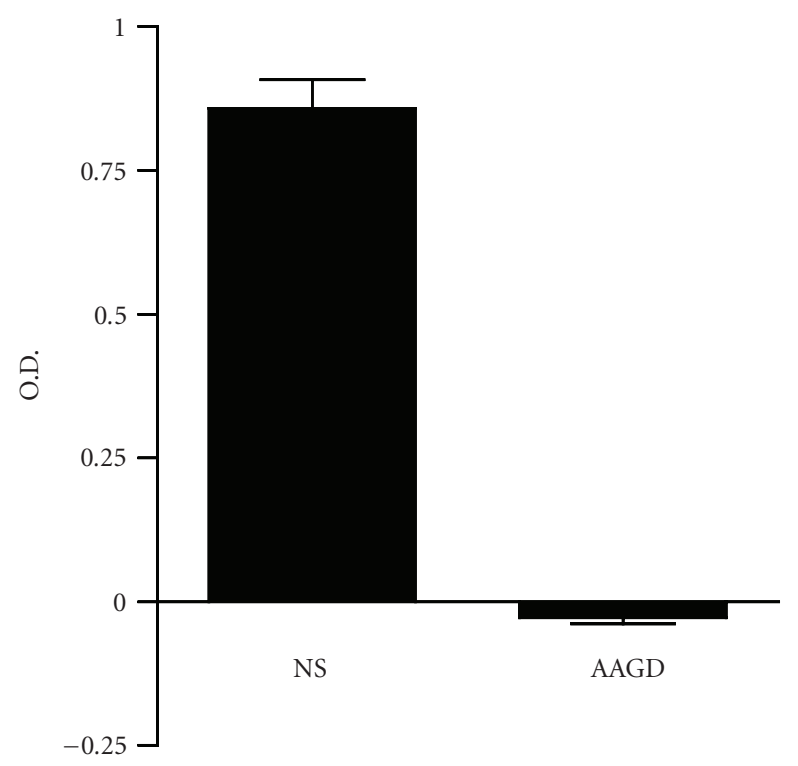

Figure 5: Comparison between normal skin (NS) and alloplastic acellular glycerolized dermis (AAGD) viability. There are no viable cells in the AAGD. The results refer to day 0.

15 colonies, with a regular shape and small dimensions (data not shown).

Immunohistochemistry and cellular viability were tested at 7 day intervals $(7,14$, and 21$)$ to verify the proliferation, differentiation and maturation of the cells on the scaffold.

\subsubsection{Histology of the AAGD+ Primary Cultured Ker-} atinocytes. A continuous double-layer of keratinocytes was clearly detected at 7 days. It was observed that the keratinocytes tended to infiltrate between the dermal papillae of the scaffold (Figure 6(a)). A pluristratified layer was present at 14 days (Figure 6(b)), which was completely differentiated at 21 days with a well-represented stratum corneum (Figure 6(c)).

\subsubsection{Histology of the AAGD+ Uncultured Keratinocytes.} There were foci and/or scattered cells at 7 days (Figure $7(\mathrm{a})$ ). The cellular morphology of the uncultured keratinocytes was elongated, with abundant cytoplasm, differing from typical basal cells in healthy skin, which are spherical and have scarce cytoplasm. A pluristratified layer invaginating into the dermis scaffold and colonising epidermal appendages (Figure 7(b)) was present at 14 days. The newly formed epithelium was completely differentiated at 21 days and showed an evident stratum corneum (Figure 7(c)).

3.3.3. Immunohistochemistry. The newly formed epithelium was analysed to evaluate the keratinocyte differentiation and maturation, compared to that of healthy skin (Figure 8). The immunohistochemistry results did not differ between primary and uncultured cells. The cytokeratin AE1/AE3 expressions were analysed by staining biosubstitute and normal skin (NS) with an antiCytokeratin AE1/AE3 MoAb.
There was a well-evidenced antibody reactivity in the NS section of all the epithelial layers (Figure 8(a)). The biosubstitute sections showed a cytoplasmic strong positivity staining in the monolayer, or cell foci at 7 days. Whilst, at 14 days the typical cytoplasmic positivity extended to the whole newly formed epithelium, involving also the pluristratified areas in the dermal papillae invagination (Figure 8(b)), which remained unaltered at 21 days.

The NS sections showed a negative antiCytokeratin 16 antibody staining (Figure 8(c)). Conversely, either the monolayer, or cell foci, stained positively in the biosubstitute biopsy sections at 7 days. A positivity in the keratinocyte cytoplasm throughout the suprabasal region with a negative epithelium basal layer was detected at 14 days (Figure 8(d)). There was a negative basal layer in the cutaneous appendages, although the more superficial layers were positive at 21 days.

The antiCytokeratin 14 antibody stained the pluristratified epithelium layers, showing a uniform positive membrane staining in NS, common to all layers (Figure 9(a)) and cutaneous appendages. Analysing our biosubstitute at 7 days, the keratinocyte cytoplasm and membrane stained positively for both the monolayer and the cell foci. At 14 and 21 days, there was cytoplasmic positive staining throughout the whole of the newly formed epithelium and cutaneous appendages (Figure 9(b)).

The E-cadherin antigen expression stained positively on the NS cell membrane at all layers (Figure 9(c)). At 7 days, the biosubstitute sections showed positivity in the keratinocyte cytoplasm, as did the basement membrane of the monolayer. Staining was observed in all epithelial layers (Figure 9(d)), also in the cutaneous appendage keratinocytes at 14 and 21 days.

3.3.4. Assessment of the Biosubstitute Viability. The viability of the newly formed epithelium was assessed by an MTT test on the biosubstitute. A normal skin sample was used as a positive control for viability. The biosubstitute was analysed at 7,14 , and 21 days after cell nebulization. The results evidenced the presence of viable cells in the biosubstitute as early as 7 days, with an increase in the positivity, which paralleled the growth and epidermal layer development over time (Figure 10). These data confirmed the results obtained by the immunohistological analysis (Figures 6 and 7).

\section{Discussion}

Tissue engineering is a multidisciplinary field focused on in vitro reconstruction of mammalian tissues. In order to allow a three-dimensional organisation of in vitro cultured cells, biocompatible scaffolds are needed.

The choice of alloplastic dermis as a scaffold is determined by the fact that it retains almost all healthy mechanical skin properties, being compact and elastic, able to take into the bed wound, providing a barrier against invading organisms: it is considered, to date, the best skin substitute available. Moreover it has been demonstrated that alloplastic dermis is a good support for the transplant of in vitro cultured keratinocytes $[15,16]$. In addition glycerolpreserved skin allografts maintain intact tissue morphology 


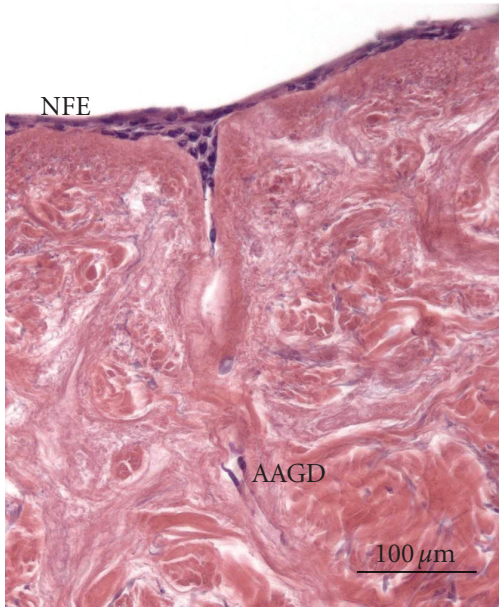

(a)

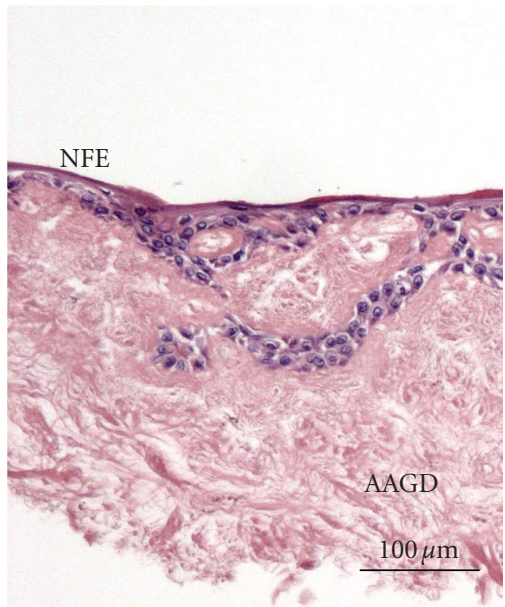

(b)

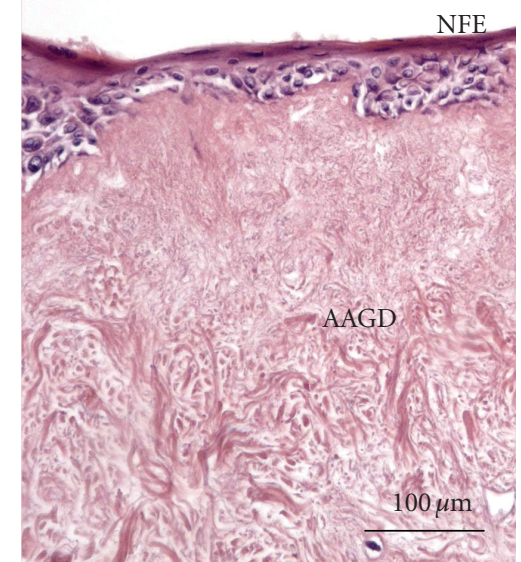

(c)

FIGURE 6: Histomorphological analysis performed on the biosubstitute sections obtained from primary culture keratinocytes at (a) 7 days, (b) 14 days, (c) 21 days post nebulization. Original magnification: 10x. AAGD: alloplastic acellular glycerolized dermis; NFE: newly formed epithelium.

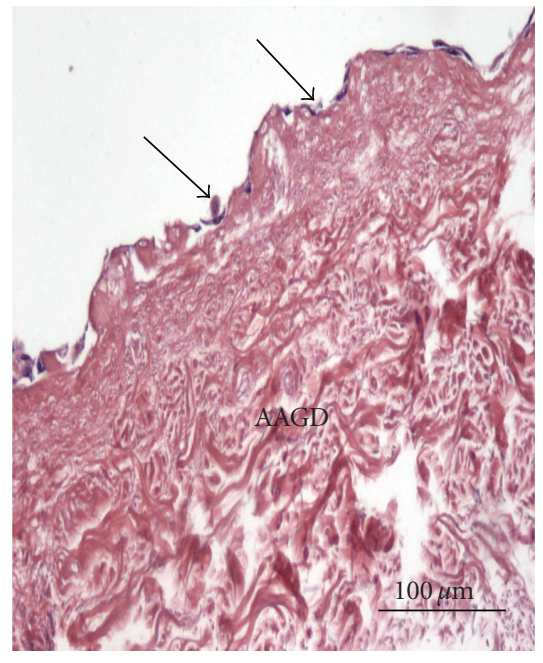

(a)

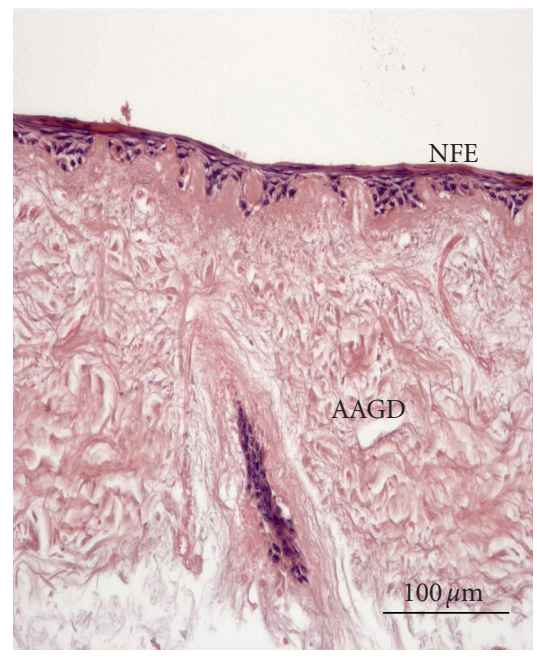

(b)

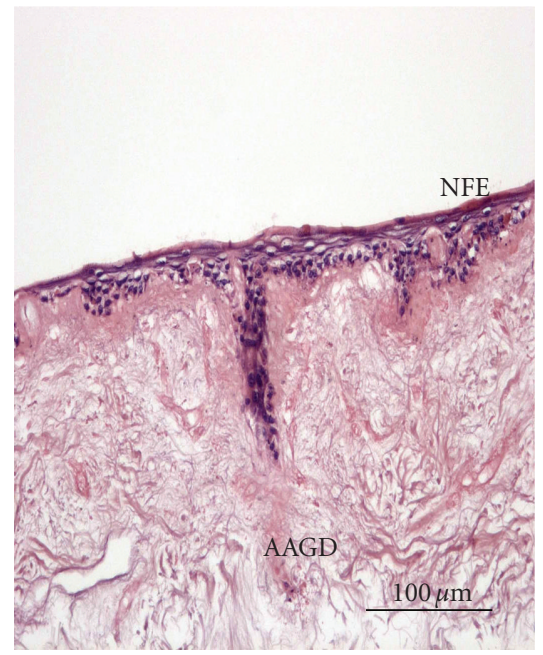

(c)

FIGURE 7: Histomorphological analysis performed on the biosubstitute sections obtained from uncultured keratinocytes at (a) 7 days, (b) 14 days, (c) 21 days post nebulization. Arrows indicate the foci and/or scattered keratinocytes. Original magnification: 10x. AAGD: alloplastic acellular glycerolized dermis; NFE: newly formed epithelium.

and show a strong decrease in tissue antigenicity compared to cryopreserved ones [11].

We hypothesised that the de-epithelialization of glycerolized skin may be an important phase in skin substitute preparation, because an adequate preservation of the basement membrane could be fundamental for both adhesion and cell growth. This prompted us to carry out research as to the best technique able to allow for a successful cell growth with a minimum manipulation.

To this aim immunohistochemical analysis was performed on the de-epithelialized alloplastic glycerolized dermis grafts, obtained by three methods, to verify the conservation state of the basement membrane. The first technique was based on dispase II enzyme used by Herson et al. [16]. Immunohistochemistry demonstrated absence of the basal membrane and, although there was a good initial keratinocyte take, at two weeks the cells began to detach from the scaffold, indicating poor cell adhesion. The second technique used trypsin treatment. Histology showed only an interrupted monolayer of cells. The epithelial removal was done manually in the third technique, with no addition of any enzyme. Histology evidenced a good cell proliferation at 7 days, which increased constantly to form multiple layers within 14 and 21 days. The last was, therefore, considered the most suitable technique for scaffold preparation, confirming the hypothesis that the basement membrane preservation 


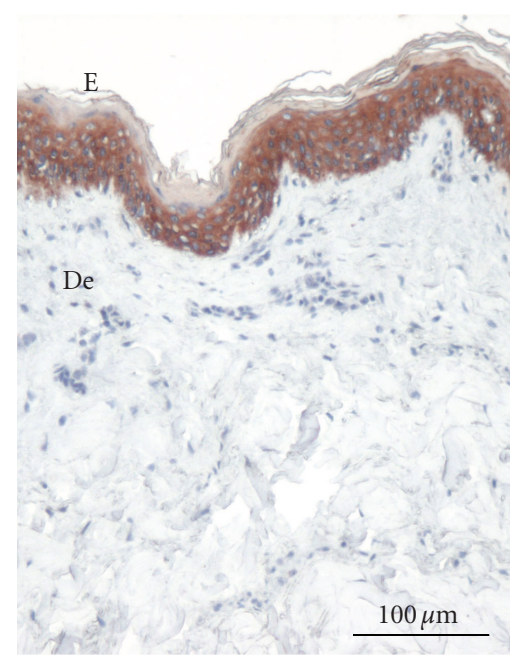

(a)

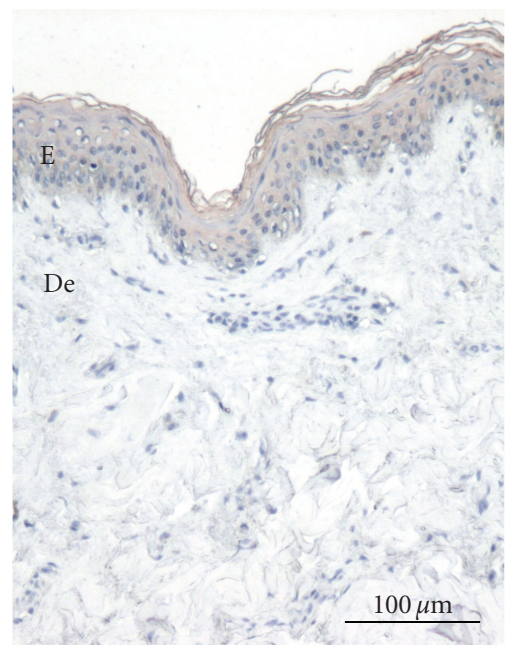

(c)

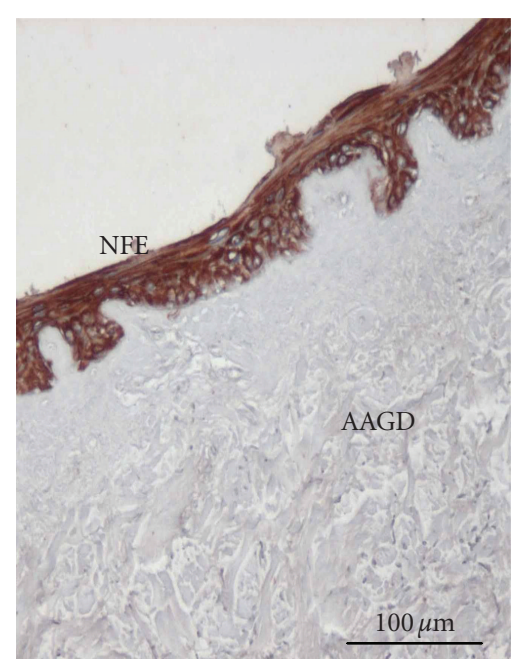

(b)

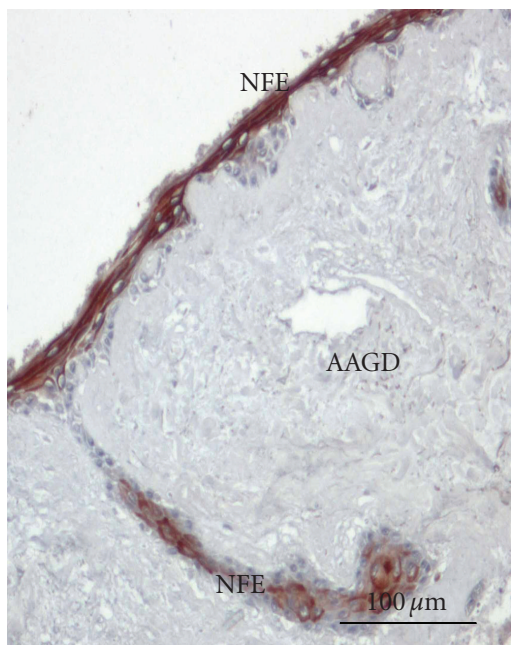

(d)

FIgURE 8: Immunohistochemical localization of human keratin in NS ((a) and (c)) and biosubstitute sections ((b) and (d)) at 14 days. ((a) and (b)): The sections were stained with cytokeratin AE1/AE3 Moab and ((c) and (d)) cytokeratin 16 Moab. Original magnification: 10x. E: epidermis, De: dermis, AAGD: alloplastic acellular glycerolized dermis, NFE: newly formed epithelium.

promotes the growth and differentiation of the keratinocytes seeded on the dermis.

The immunogenicity of the scaffold was then evaluated. Although the morphology and structure of the dermis is maintained when skin is preserved in glycerol at $85 \%$, it looses cell viability and is, therefore, scarcely antigenic [11, 17]. Indeed, a low immunogenicity is fundamental for the preparation of cutaneous substitutes, which must, not only adhere to the lesion bed, but must also avoid triggering a rejection. The scaffold was analysed for cell immunogenicity using specific markers for lymphocytes, macrophages and dendritic cells. Immunohistochemistry demonstrated that lymphocytes, macrophages or dendritic cells in the AADG after five days of culture were absent. Scaffold viability tests based on MTT salts were negative, indicating that no viable cells were present in the scaffold.
Because engineered tissues are designed for clinical applications in humans, a major problem is the possible contamination of cocultures and tissues by xenogenic molecules used to grow stem cells in vitro. The protocols that are commonly applied to generate epidermal equivalents in vitro in fact require the use of irradiated murine fibroblasts (3T3 J2) as a feeder layer for keratinocytes. In this study we report a simple procedure for growing human keratinocytes, isolated from adult skin, to generate an epidermal construct on AADG alone. In fact initially a feeder layer of irradiated murine 3T3 fibroblasts was used, followed by the culture of primary keratinocytes on the scaffold. When the keratinocytes were cultured directly on alloplastic dermis, no difference in keratinocyte proliferation was observed (data not shown). It was, therefore, decided to avoid the use of murine cells for the preparation of the biosubstitute. 


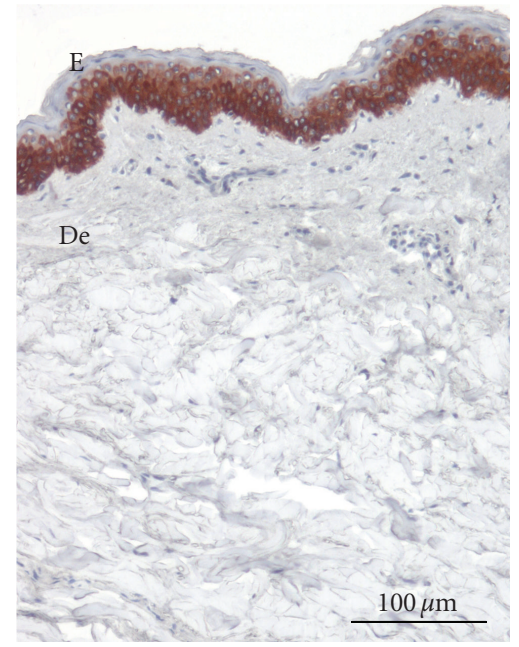

(a)

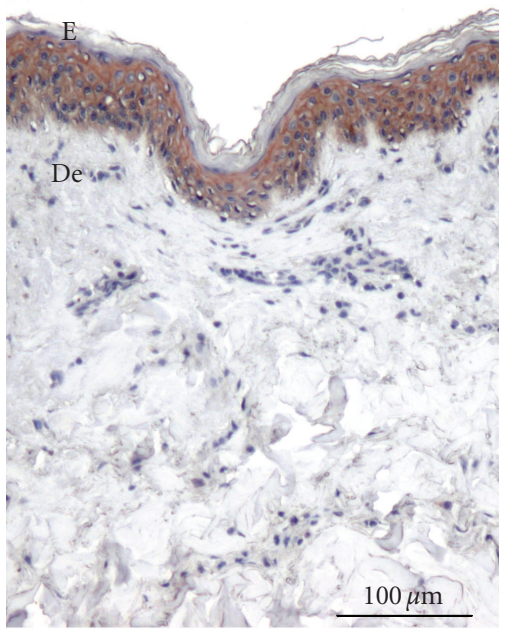

(c)

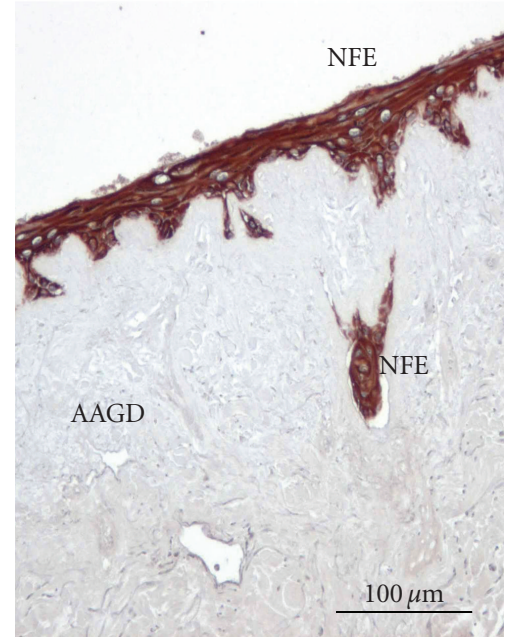

(b)

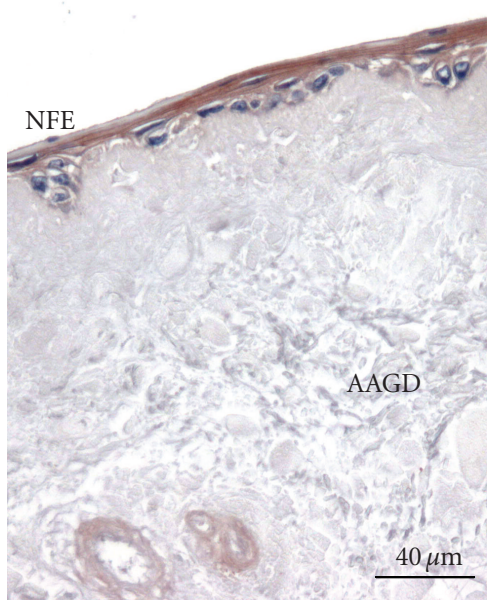

(d)

FIGURE 9: Immunohistochemical localization of human keratin in NS ((a) and (c)) and biosubstitute ((b) and (d)) at 14 days. ((a) and (b)): The sections were stained with cytokeratin 14 Moab and ((c) and (d)) E-Cadherin Moab. Original magnification: 10x ((a), (b), and (c)) 20x (d). E: epidermis, De: dermis, AAGD: alloplastic acellular glycerolized dermis, NFE: newly formed epithelium.

Morphological, immunohistochemical and cytokinetic features of epithelial colonies grown on the AADG layer were typical of keratinocytes and were comparable with those reported for keratinocytes grown on a feeder layer. In fact histology showed a good keratinocyte growth at 7 days which formed a continuous pluristratified layers within 14/21 days, and at the same time the viability increased constantly in direct proportion to the days of culture.

The quality of the newly formed epithelium was evaluated by specific antibodies directed against cytokeratins and specific markers of keratinocyte differentiation, which have a varied distribution in cutaneous tissue.

The analysis of cytokeratin 14 shows a distribution similar to healthy skin, confirming the ability of cultured cells to form a stratified epithelium.

Cytokeratin 16 expression is upregulated during processes of keratinocyte activation, which occur during wound healing and is markedly overexpressed in epithelia that display abnormal differentiation, such as the psoriatic epidermis [18-20], while, according to literature [21], is downregulated in NS.

The biosubstitute sections showed a marked cytoplasmic positivity to CK16 when epithelial cells grow, in the initial proliferative phase, as a monolayer. On the opposite, when epithelial cells were pluristratified, CK16 positivity was localised only in the most superficial layers, as in epithelial regenerative processes [22].

The anticytokeratin AE1/AE3 MoAb recognises most human cytokeratin and identifies epithelial cells in stratified and simple layers, and is expressed in the stratum corneum and the hyperproliferating keratinocytes [23]. All the corneous layer and some keratinocyte foci were positive at the membrane level in the NS sections, in agreement with literature. The epithelium of biosubstitute sections was positive 


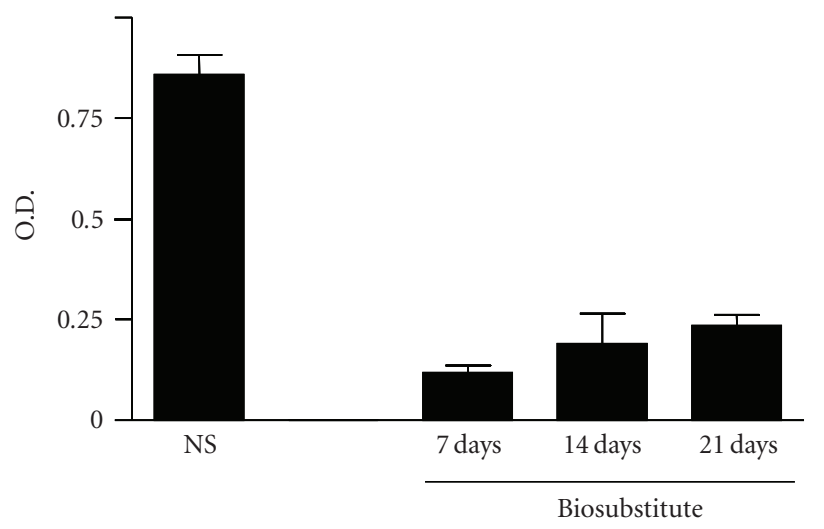

Figure 10: The MTT test was used to assess tissue viability in normal skin (NS) and biosubstitute. A NS sample was used as a positive control. The viability of skin substitute was analyzed at 7 , 14 and 21 days after cell nebulization. A constant increase in the cell viability was observed over time, parallel to the growth and epidermal layer development.

at the cytoplasmic level, both in the monostratified and pluristratified areas. The cells of dermal papillae and neocolonized appendages were also positive, as was expected, by regenerating epithelium.

The antiE-cadherin antibody recognizes the transmembrane molecules that connect to the actin cytoskeleton via catenins. The E-cadherin has a significant function in the intercellular adhesion of epithelial cells as it establishes epithelial polarization, glandular differentiation and stratification. The NS epithelium cell membrane was positive in all layers, as reported in literature [24]. The antibody staining was evident both on membrane and cytoplasm in all the epithelial strata of the biosubstitute sections, demonstrating that the newly formed epithelium keratinocytes had a polarization and differentiation grade comparable to that of normal epithelial cells. The E-cadherin antigen expression was negative in the dermal papillae, where the keratinocytes were still undergoing the process of migration, in agreement with their differentiated state.

Taken together our immunohistochemical results suggest that the newly formed epithelium of the biosubstitute was comparable to an epidermis in active regeneration as is observed during the process of wound healing, where the keratinocytes are in an activated state.

In this paper two different keratinocytes suspensions were used: cultured and uncultured cells. In fact also uncultured cells were sprayed directly onto the alloplastic dermis in order to improve the timing of the cells growth. The biosubstitutes were then monitored to asses any growth differences between those of the biosubstitute obtained with the cultured primary and uncultured cells. The most significant differences were evident at 7 days where there was no double-layer in the biosubstitute obtained with uncultured cells, but only cellular foci that form a multilayer at 14 days. At 21 days, the newly formed epithelium, in both cases, was completely differentiated and showed an evident stratum corneum. Moreover these data confirmed the results obtained by the MTT analysis [25].

\section{Conclusion}

The promising preliminary results obtained in this paper indicate that the newly formed skin has all the differentiation and maturation characteristics of a good cutaneous substitute for clinical application on burn patients and/or other subjects with serious skin diseases.

\section{Acknowledgments}

This paper was supported by funds from the Compagnia di San Paolo, Turin, Italy, Fondazione CRT, Turin Italy, Eni Italgas, and Piedmont Foundation of Studies and Research on Burns (President, Simone Teich Alasia). Irene Cambieri, Mara Fumagalli, Alessia Ostorero were supported by a fellowship from the Compagnia di San Paolo. Daniela Alotto and Stefania Casarin were supported by a fellowship from the Piedmont Foundation of Studies and Research on Burns. The authors would also like to thank Mrs Barbara Wade for her linguistic advice.

\section{References}

[1] A. T. N. Truong, A. Kowal-Vern, B. A. Latenser, D. E. Wiley, and R. J. Walter, "Comparison of dermal substitutes in wound healing utilizing a nude mouse model," Journal of Burns and Wounds, vol. 4, p. e4, 2005.

[2] V. Ronfard, J.-M. Rives, Y. Neveux, H. Carsin, and Y. Barrandon, "Long-term regeneration of human epidermis on third degree burns transplanted with autologous cultured epithelium grown on a fibrin matrix," Transplantation, vol. 70, no. 11, pp. 1588-1598, 2000.

[3] H. Green, O. Kehinde, and J. Thomas, "Growth of cultured human epidermal cells into multiple epithelia suitable for grafting," Proceedings of the National Academy of Sciences of the United States of America, vol. 76, no. 11, pp. 5665-5668, 1979.

[4] N. E. O'Connor, J. B. Mulliken, and S. Banks-Schlegel, "Grafting of burns with cultured epithelium prepared from autologous epidermal cells," The Lancet, vol. 317, no. 8211, pp. 75-78, 1981.

[5] G. G. Gallico III, N. E. O’Connor, and C. C. Compton, "Permanent coverage of large burn wounds with autologous cultured human epithelium," The New England Journal of Medicine, vol. 311, no. 7, pp. 448-451, 1984.

[6] L. Donati, G. Magliacani, M. Bormioli, M. Signorini, and F. W. Baruffaldi Preis, "Clinical experiences with keratinocyte grafts," Burns, vol. 18, supplement 1, pp. S19-S20, 1992.

[7] H. Carsin, P. Ainaud, H. Le Bever et al., "Cultured epithelial autografts in extensive burn coverage of severely traumatized patients: a five year single-center experience with 30 patients," Burns, vol. 26, no. 4, pp. 379-387, 2000.

[8] C. Cuono, R. Langdon, and J. McGuire, "Use of cultured epidermal autografts and dermal allografts as skin replacement after burn injury," The Lancet, vol. 17, no. 8490, pp. 11231124, 1986.

[9] J. Schlabe, C. Johnen, R. Schwartlander et al., "Isolation and culture of different epidermal and dermal cell types from human scalp suitable for the development of a therapeutical cell spray," Burns, vol. 34, no. 3, pp. 376-384, 2008. 
[10] C. Johnen, B. Hartmann, I. Steffen et al., "Skin cell isolation and expansion for cell transplantation is limited in patients using tobacco, alcohol, or are exhibiting diabetes mellitus," Burns, vol. 32, no. 2, pp. 194-200, 2006.

[11] C. D. Richters, M. J. Hoekstra, J. Van Baare, J. S. Du Pont, and E. W. A. Kamperdijk, "Morphology of glycerol-preserved human cadaver skin,” Burns, vol. 22, no. 2, pp. 113-116, 1996.

[12] J. G. Rheinwald and H. Green, "Serial cultivation of strains of human epidermal keratinocytes: the formation of keratinizing colonies from single cells," Cell, vol. 6, no. 3, pp. 331-334, 1975.

[13] D. Alotto, S. Ariotti, S. Graziano et al., "The role of quality control in a skin bank: tissue viability determination," Cell and Tissue Banking, vol. 3, no. 1, pp. 3-10, 2002.

[14] C. E. M. Griffiths, J. N. W. N. Barker, S. Kunkel, and B. J. Nickoloff, "Modulation of leucocyte adhesion molecules, a Tcell chemotaxin (IL-8) and a regulatory cytokine (TNF- $\alpha$ ) in allergic contact dermatitis (rhus dermatitis)," British Journal of Dermatology, vol. 124, no. 6, pp. 519-526, 1991.

[15] I. McKay, B. Woodward, K. Wood, H. A. Navsaria, H. Hoekstra, and C. Green, "Reconstruction of human skin from glycerol-preserved allodermis and cultured keratinocyte sheets," Burns, vol. 20, no. 1, pp. S19-S22, 1994.

[16] M. R. Herson, M. B. Mathor, S. Altran, V. L. Capelozzi, and M. C. Ferreira, "In vitro construction of a potential skin substitute through direct human keratinocyte plating onto decellularized glycerol-preserved allodermis," Artificial Organs, vol. 25, no. 11, pp. 901-906, 2001.

[17] J. Van Baare, E. E. J. Ligtvoet, and E. Middelkoop, "Microbiological evaluation of glycerolized cadaveric donor skin," Transplantation, vol. 65, no. 7, pp. 966-970, 1998.

[18] P. A. Coulombe, N. S. Bravo, R. D. Paladini, D. Nguyen, and K. Takahashi, "Overexpression of human keratin 16 produces a distinct skin phenotype in transgenic mouse skin," Biochemistry and Cell Biology, vol. 73, no. 9-10, pp. 611-618, 1995.

[19] R. D. Paladini, K. Takahashi, N. S. Bravo, and P. A. Coulombe, "Onset of re-epithelialization after skin injury correlates with a reorganization of keratin filaments in wound edge keratinocytes: defining a potential role for keratin 16," Journal of Cell Biology, vol. 132, no. 3, pp. 381-397, 1996.

[20] P. A. Coulombe, "Towards a molecular definition of keratinocyte activation after acute injury to stratified epithelia," Biochemical and Biophysical Research Communications, vol. 236, no. 2, pp. 231-238, 1997.

[21] A. K. Smiley, J. M. Klingenberg, B. J. Aronow, S. T. Boyce, W. J. Kitzmiller, and D. M. Supp, "Microarray analysis of gene expression in cultured skin substitutes compared with native human skin," Journal of Investigative Dermatology, vol. 125, no. 6, pp. 1286-1301, 2005.

[22] I. M. Leigh, H. Navsaria, P. E. Purkis, I. A. McKay, P. E. Bowden, and P. N. Riddle, "Keratins (K16 and K17) as markers of keratinocyte hyperproliferation in psoriasis in vivo and in vitro," British Journal of Dermatology, vol. 133, no. 4, pp. 501$511,1995$.

[23] R. A. Weiss, R. Eichner, and T. T. Sun, "Monoclonal antibody analysis of keratin expression in epidermal diseases: a 48- and 56-kdalton keratin as molecular markers for hyperproliferative keratinocytes," Journal of Cell Biology, vol. 98, no. 4, pp. 1397-1406, 1984.

[24] M. E. Bracke, F. M. Van Roy, and M. M. Mareel, "The Ecadherin/catenin complex in invasion and metastasis," Current Topics in Microbiology and Immunology, vol. 213, part 1, pp. 123-161, 1996.
[25] C. Castagnoli, D. Alotto, I. Cambieri et al., "Evaluation of donor skin viability: fresh and cryopreserved skin using tetrazolioum salt assay," Burns, vol. 29, no. 8, pp. 759-767, 2003. 


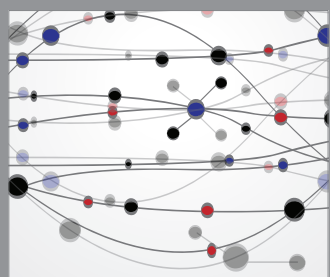

The Scientific World Journal
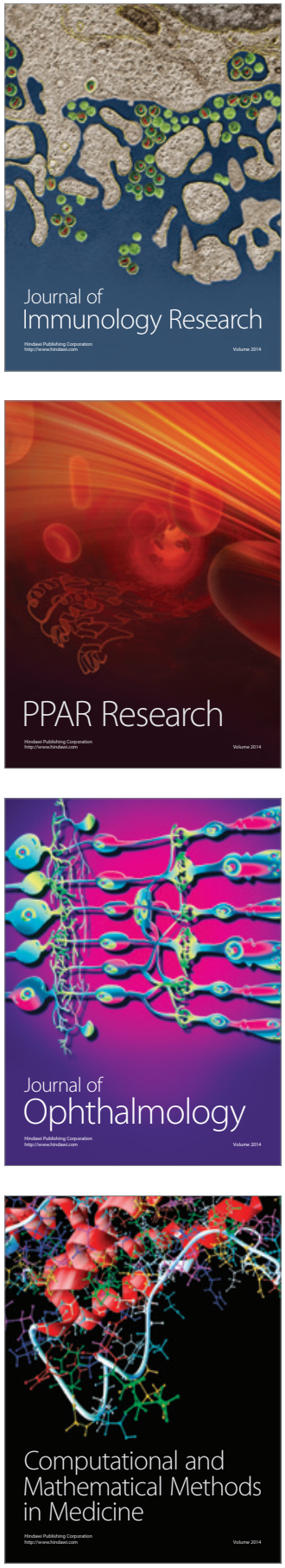

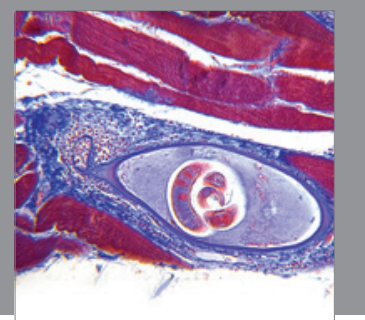

Gastroenterology

Research and Practice
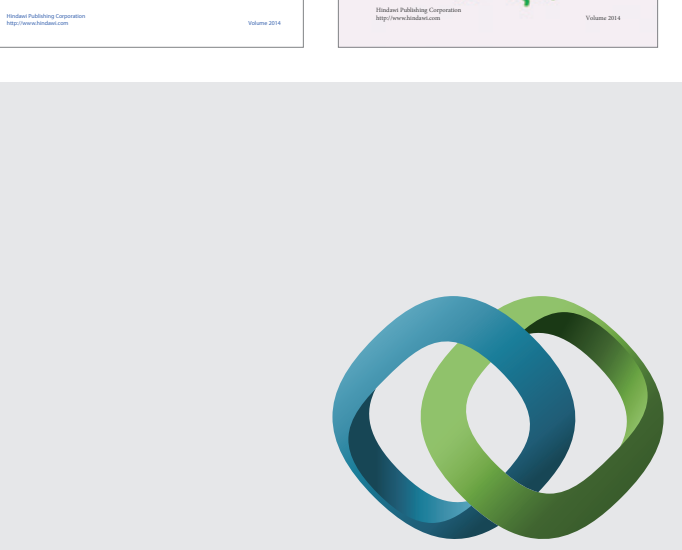

\section{Hindawi}

Submit your manuscripts at

http://www.hindawi.com
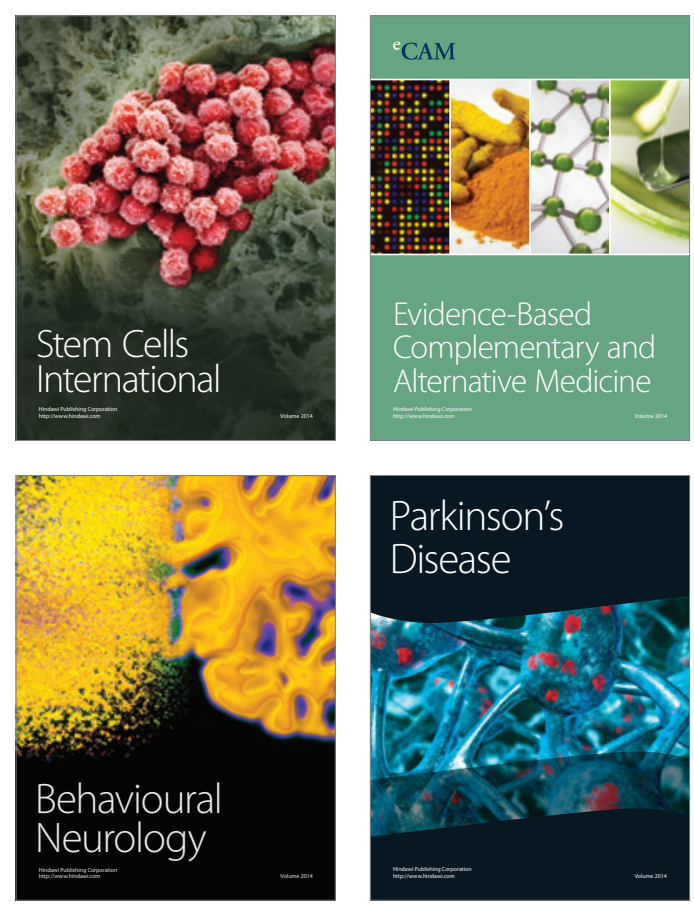

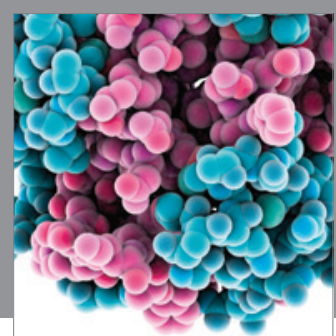

Journal of
Diabetes Research

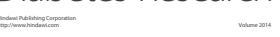

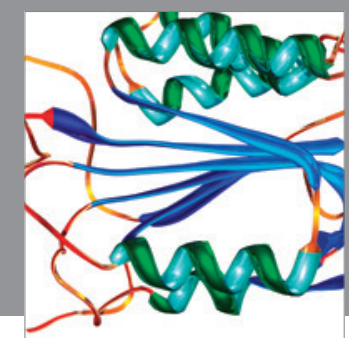

Disease Markers
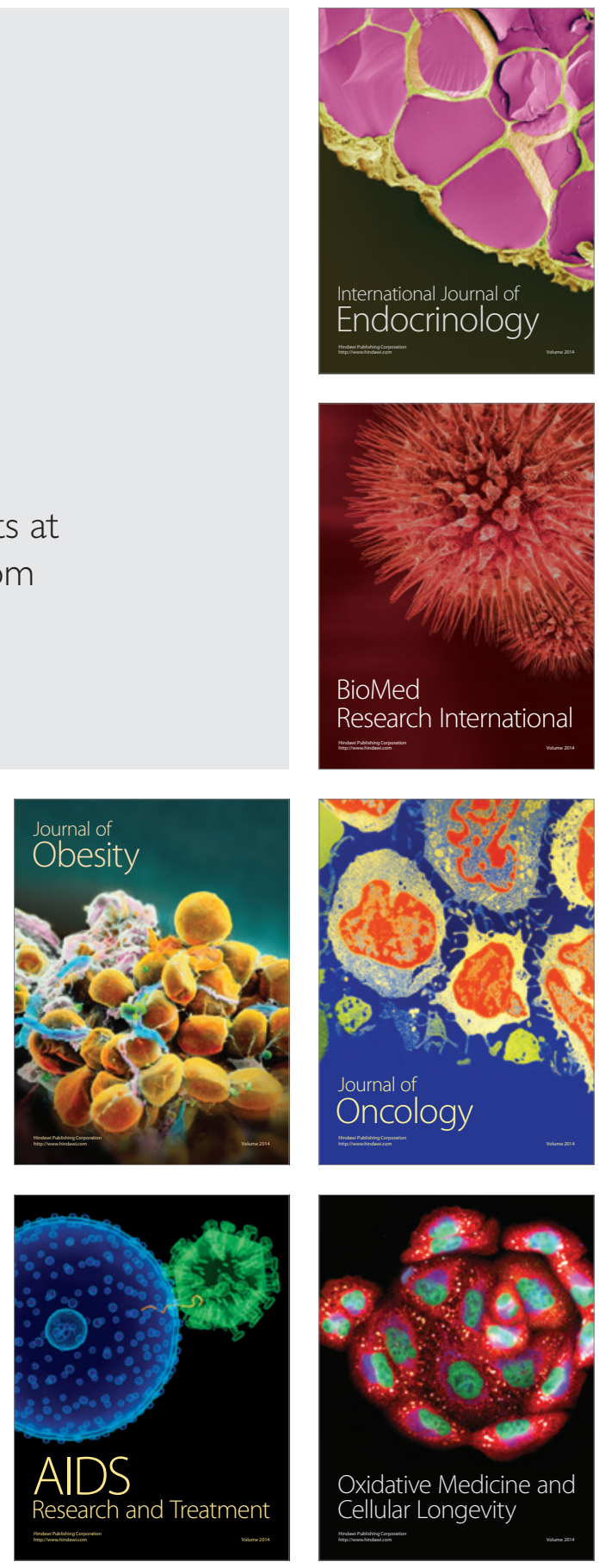